\title{
Oxygen Concentration-Dependent Oxidative Stress Levels in Rats
}

\author{
Fumiko Nagatomo, ${ }^{1}$ Hidemi Fujino, ${ }^{2}$ Hiroyo Kondo, ${ }^{3}$ and Akihiko Ishihara ${ }^{1}$ \\ ${ }^{1}$ Laboratory of Cell Biology and Life Science, Graduate School of Human and Environmental Studies, Kyoto University, \\ Kyoto 606-8501, Japan \\ ${ }^{2}$ Department of Rehabilitation Science, Kobe University Graduate School of Health Sciences, Kobe 654-0142, Japan \\ ${ }^{3}$ Department of Food Sciences and Nutrition, Nagoya Women's University, Nagoya 467-8610, Japan \\ Correspondence should be addressed to Akihiko Ishihara, ishihara.akihiko.8s@kyoto-u.ac.jp
}

Received 19 April 2012; Revised 5 August 2012; Accepted 8 August 2012

Academic Editor: Jose Luis Martin-Ventura

Copyright ( 2012 Fumiko Nagatomo et al. This is an open access article distributed under the Creative Commons Attribution License, which permits unrestricted use, distribution, and reproduction in any medium, provided the original work is properly cited.

\begin{abstract}
Introduction. We determined derivatives of reactive oxygen metabolites (dROMs) as an index of oxidative stress level (oxidant capacity) and biochemical antioxidant potential (BAP) as an index of antioxidant capacity in rats exposed to different oxygen concentrations. Methods. Male Wistar rats were exposed to $14.4 \%, 20.9 \%, 35.5 \%, 39.8 \%, 62.5 \%$, and $82.2 \%$ oxygen at 1 atmosphere absolute for $24 \mathrm{~h}$. Serum levels of dROMs and BAP were examined by using a free radical and antioxidant potential determination device. The morphological characteristics of red blood cells were examined by phase contrast microscopy. Results. There were no differences in the levels of dROMs in rats exposed to $14.4 \%, 20.9 \%$, and 35.5\% oxygen. However, the levels of dROMs increased in the rats exposed to $39.8 \%$ and $62.5 \%$ oxygen. The levels of dROMs were the highest in the rats exposed to $82.2 \%$ oxygen. There were no differences in the levels of BAP with respect to the oxygen concentration. Morphological changes in the red blood cells induced by oxidative attack from reactive oxygen species were observed in the rats exposed to $39.8 \%, 62.5 \%$, and $82.2 \%$ oxygen. Conclusion. Our results suggest that exposure to oxygen concentrations higher than $40 \%$ for $24 \mathrm{~h}$ induces excessive levels of oxidative stress in rats.
\end{abstract}

\section{Introduction}

Supplemental oxygen is used in treatment and as a countermeasure for acute and chronic diseases. When pilots of unpressurized aircrafts fly to areas at high altitudes, when climbers ascend high-altitude peaks and outpace their ability to acclimatize, or when divers inhaling compressed air return to the surface, the external pressure on the body decreases and the dissolved inert gases come out of solution in the form of bubbles in the body on depressurization $[1,2]$. The resulting decompression sicknesses and air embolisms are initially treated by inhalation of oxygen-enriched air or exposure to mild hyperbaric oxygen at 1.25 atmospheres absolute (ATA) until hyperbaric oxygen therapy (100\% oxygen delivered at 2-3 ATA) is administered [3-6]. Hypoxic or breathless patients with chronically obstructive pulmonary disease (COPD), who have low levels of oxygen in their blood, require oxygen at concentrations greater than that in room air to achieve arterial oxygen saturations between $88 \%$ and $92 \%[7]$.

Oxygen therapy with or without pressure is associated with the risk of oxygen toxicity and excessive oxidative stress. Oxidative stress plays as key role in the pathogenesis of many diseases and their complications; the generation of free radicals and increased levels of oxidative stress are associated with atherosclerosis, cataract, retinopathy, myocardial infarction, hypertension, diabetes, renal failure, and uremia [8-10]. However, there are no data available on changes in the oxidative stress level and antioxidant capacity after exposure to different concentrations of oxygen. The analytical measurement of oxidative stress markers has been difficult because of the short half-life and high reactivity of the majority of reactive oxygen species and the applicability of measurement methods [11]. Blood samples are the appropriate biological materials for assessing the status of oxidants and antioxidants. A unique system for the 
evaluation of oxidative stress levels and antioxidant capacity in the blood has been developed [12]. This evaluation approach is based on the free radical analytical system that mainly analyzes lipid hydroperoxides, which are relatively stable in the blood. This system has been used for both animal and human sera, which confirms its applicability [13-15].

In this study, we examined the derivatives of reactive oxygen metabolites (dROMs) as an index of oxidative stress levels (oxidant capacity) and the biochemical antioxidant potential (BAP) as an index of antioxidant capacity in rats exposed to different concentrations of oxygenat 1 ATA for $24 \mathrm{~h}$.

\section{Materials and Methods}

2.1. Experimental Animals. All experimental and animal care procedures were performed in accordance with the guidelines stated in the Guide for the Care and Use of Laboratory Animals issued by the Institutional Animal Experiment Committee of Kyoto University (Kyoto, Japan).

2.2. Exposure to Different Concentrations of Oxygen. Tenweek-old male Wistar rats weighing between $200 \mathrm{~g}$ and $226 \mathrm{~g}$ were divided into 6 groups ( $n=5$ for each group). The individual groups were exposed to air containing low or high concentration of oxygen in a chamber $(75 \times 130 \times 85 \mathrm{~cm})$ at 1 ATA for $24 \mathrm{~h}$ by using a low-oxygen inhaler (Terucom Corp., Yokohama, Japan) or an oxygen concentrator (Ikiken Corp., Sayama, Japan), respectively. When the air chamber contained less or more than $20.9 \%$ oxygen, air with 2 different concentrations of oxygen from 2 tubes was transported to the aspirator: one tube contained normal air $(20.9 \%$ oxygen) and the other tube had air containing $13 \%$ or $84 \%$ oxygen, which was procured from the low-oxygen inhaler or the oxygen concentrator, respectively. Then, the aspirator pumped mixed air into the chamber at the rate of $1 \mathrm{~L} / \mathrm{min}$. The low or high oxygen concentration in the chamber was adjusted by separately regulating the air flow from these 2 tubes. Normal air was transported by only 1 tube, while the air containing $20.9 \%$ oxygen was retained in the chamber. The oxygen concentration in the chamber was determined by using an oxygen monitor $\left(\mathrm{Max} \mathrm{O}_{2}+\mathrm{AE}\right.$; Maxtec Inc., UT, USA) attached to the chamber. The rats were maintained in individual, uniformly sized standard cages $(30 \times 40 \times 20 \mathrm{~cm})$ in the chamber. The room was maintained at $22 \pm 2{ }^{\circ} \mathrm{C}$ with $45 \%-55 \%$ relative humidity. Food and water were provided ad libitum.

2.3. Measurements of $d R O M$ s and BAP. The levels of dROMs and BAP were determined after the rats were exposed to different concentrations of oxygen. Blood samples were obtained from the tail of fully conscious rats and evaluated photometrically. A free radical and antioxidant potential determination device (Free Radical Analytical System 4; Health \& Diagnostics, Grosseto, Italy) was used to automatically measure the levels of dROMs and BAP.
The dROMs are used as an index to determine the level of oxidative stress (oxidant capacity) by measuring the amount of organic hydroperoxide ( $\mathrm{ROOH})$ converted into radicals that oxidize $N, N$-diethyl- $p$-phenylenediamine $[12,16]$. The levels of dROMs were expressed in Carr units ( $1 \mathrm{U}$-Carr corresponds to $0.08 \mathrm{mg}$ hydroperoxide $/ 100 \mathrm{~mL}$ $\mathrm{H}_{2} \mathrm{O}_{2}$ ). The BAP is used as an index to determine the biological antioxidant capacity and is measured on the basis of the capacity of the plasma sample to reduce ferric ions to ferrous ions. After blood samples were obtained, the rats were killed by intraperitoneal overdose of sodium pentobarbital.

2.4. Red Blood Cell Morphology. Using blood samples, the morphological profiles of red blood cells were observed by phase contrast microscopy (Nikon 80iF-PH-15; Tokyo, Japan).

2.5. Statistics. Means and standard deviations were calculated from the individual values by using standard procedures. One-way analysis of variance (ANOVA) was used to evaluate the mean differences among the 6 groups. When ANOVA analyses revealed significant differences in mean values, the groups were further compared using Scheffé's post hoc tests. A probability level of 0.05 was considered significant.

\section{Results}

The levels of dROMs in rats exposed to $39.8 \%$ and $62.5 \%$ oxygen were higher than those in the rats exposed to $14.4 \%$, $20.9 \%$, and $35.5 \%$ oxygen (Figure $1(\mathrm{a})$ ). The level of dROMs in the rats exposed to $82.2 \%$ oxygen was the highest among the 6 groups. There were no differences in the levels of BAP among the 6 groups (Figure 1(b)). Morphological changes in red blood cells were observed in the rats exposed to $39.8 \%$, $62.5 \%$, and $82.2 \%$ oxygen (Figure 2 ).

\section{Discussion}

4.1. Exposure to Low Concentration of Oxygen. Acclimatization at high altitude results in changes in the respiratory, cardiovascular, and hematologic systems, which enhance oxygen delivery to the cells and tissues [17]. Decompression sickness occurs between initial hypoxic conditions and the onset of acclimatization, and the incidence and severity of the sickness depend on the rate of ascent, the altitude attained, and physiological susceptibility of individuals [5].

There is little data available regarding the oxidative stress level under low concentrations of oxygen. In this study, we examined the oxidative stress levels in rats exposed to low concentrations of oxygen. In rats exposed to $14.4 \%$ oxygen for $24 \mathrm{~h}$, which is equivalent to the oxygen concentration at $3500 \mathrm{~m} / 11500$ feet altitude, no change was observed in the oxidative stress level (Figure 1(a)). Therefore, we conclude that low concentrations of oxygen do not induce excessive oxidative stress. 


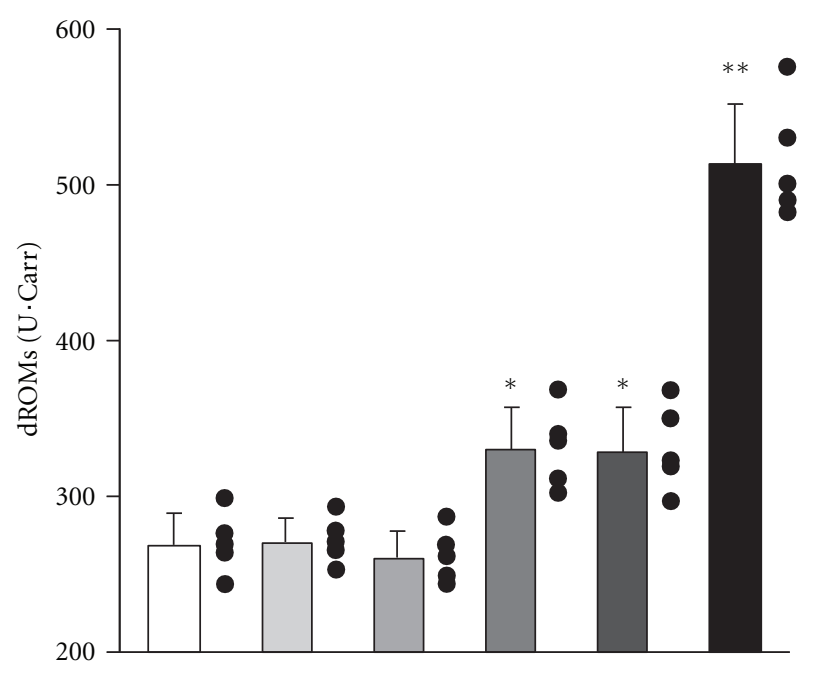

(a)

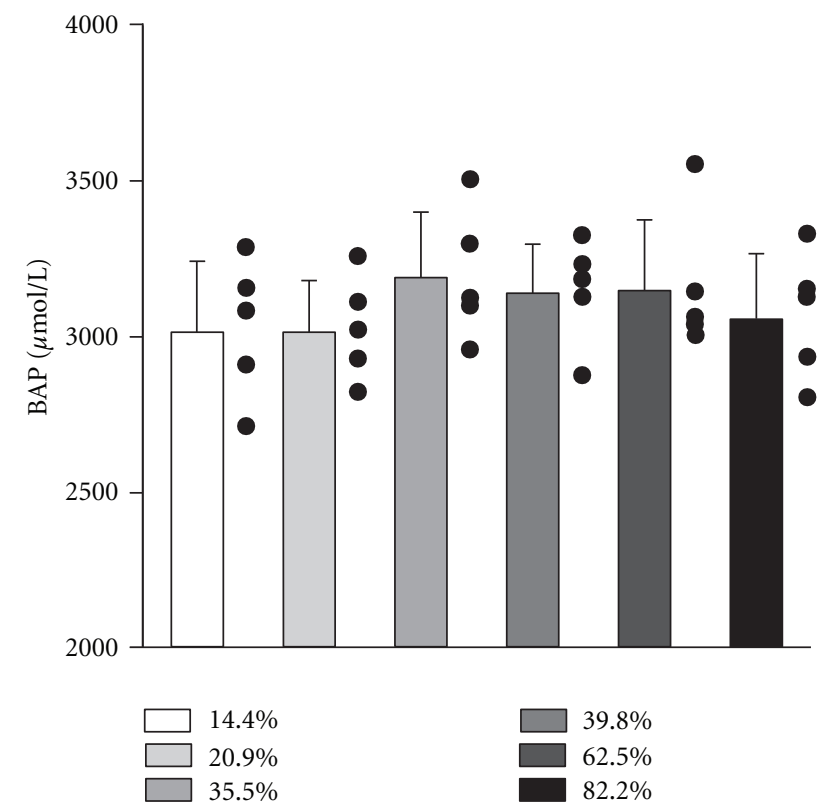

(b)

FIgURE 1: Levels of derivatives of reactive oxygen metabolites (a) and biochemical antioxidant potential (b) of rats after exposure to different concentrations of oxygen for $24 \mathrm{~h}$. Values are expressed as the mean and standard deviation ( $n=5$ for each group). Five dots on the right side of the bar are the individual values of rats in each group. dROMs: derivatives of reactive oxygen metabolites; BAP: biochemical antioxidant potential. ${ }^{*} P<0.05$ compared with values of $14.4 \%, 20.9 \%$, and $35.5 \%$ oxygen; ${ }^{* *} P<0.05$ compared with values of $14.4 \%, 20.9 \%, 35.5 \%, 39.8 \%$, and $62.5 \%$ oxygen.

4.2. Exposure to High Concentration of Oxygen. Hyperbaric oxygen at 2-3 ATA with 100\% oxygen induces acute changes, such as increased blood pressure and further reduction in heart rate [18], and causes chronic diseases like cataract formation [19-22]; hyperbaric oxygen is generally safe when pressures do not exceed 3 ATA and the length of treatment is less than $120 \mathrm{~min}[23,24]$. However, hyperbaric oxygen has been reported to increase the levels of reactive oxygen species [25-27].

Patients with COPD inhale high concentrations of oxygen, generally up to 50\% oxygen (fraction of inspired oxygen, $\mathrm{FIO}_{2}$ ) when the partial pressure of oxygen in the arterial blood $\left(\mathrm{PaO}_{2}\right)$ is below $55 \mathrm{mmHg}$; these patients occasionally exhibit voluntary respiration failure, consciousness disturbance, and atelectasis when inhaling high concentrations of oxygen.

Oxygen therapy with or without pressure might induce excessive levels of oxidative stress. Excessive levels of oxidative stress are associated with many diseases, including atherosclerosis, cataract, retinopathy, myocardial infarction, hypertension, renal failure, and uremia [8-10]. In this study, we examined the oxidative stress levels in rats exposed to high concentrations of oxygen.

No change in the oxidative stress level was observed at $35.5 \%$ oxygen for $24 \mathrm{~h}$ (Figure $1(\mathrm{a})$ ). We previously examined the effects of mild hyperbaric oxygen at 1.25 ATA with $36 \%$ oxygen on the neuromuscular system, including spinal motoneurons and their innervating muscle fibers [28, 29], type 2 diabetes [30-33], hypertension [34], type II collagen-induced arthritis [35], age-related decline in muscle oxidative capacity [36], and diabetes-induced cataracts [37] in mice and rats. Therefore, the data observed in this study suggest that mild hyperbaric oxygen at 1.25 ATA with $36 \%$ oxygen is effective for the inhibition and improvement of many metabolic diseases [28-37], without producing excessive levels of oxidative stress.

In contrast, $39.8 \%$ and $62.5 \%$ oxygen for $24 \mathrm{~h}$ induced excessive levels of oxidative stress, and the level of dROMs was the highest at $82.2 \%$ oxygen (Figure 1(a)). Oxidative stress level increases when the production of reactive oxygen species is markedly greater than the intrinsic antioxidant defenses. Patients with COPD inhale high concentrations of oxygen, and the possibility of accumulating excessive levels of oxidative stress will be greater when the inhaled oxygen concentration $\left(\mathrm{FIO}_{2}\right)$ is high. Therefore, we conclude that exposure to $40 \%$ oxygen for $24 \mathrm{~h}$ is a threshold for inducing an excessive level of oxidative stress.

In our previous study [15], we observed that obese rats with metabolic syndrome accompanied by insulin resistance, impaired glucose metabolism, and dyslipidemia had lower levels of BAP than normal Wistar rats. In addition, we observed that mild hyperbaric exposure at 1.25 ATA with $36 \%$ oxygen improves the levels of BAP in rats with hypertension [34]. These studies $[15,34]$ suggest that changes in the levels of BAP are reflected as an index of antioxidant capacity. In this study, we expected that exposure to higher concentrations of oxygen would decrease the levels of BAP compared with exposure to $20.9 \%$ oxygen because the levels of dROMs were increased by exposure to $39.8 \%, 62.5 \%$, and $82.2 \%$ oxygen (Figure 1(a)). However, there was no difference in the levels of BAP among different concentrations of oxygen (Figure 1(b)). These results suggest that the antioxidant capacity is not affected by both low and high concentrations of oxygen, and thus, the antioxidant capacity did not change, at least after $24 \mathrm{~h}$. However, we did not examine antioxidant enzyme levels of rats exposed to different concentrations 


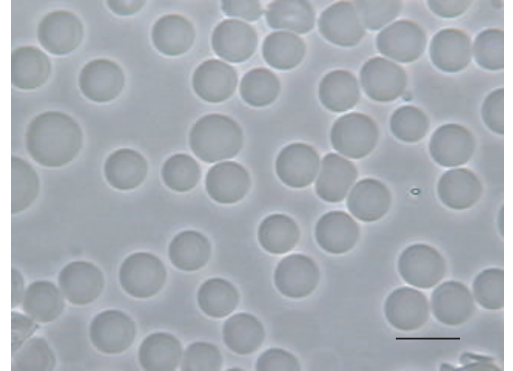

(a)

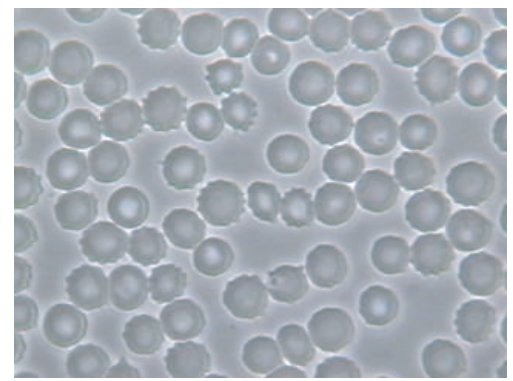

(d)

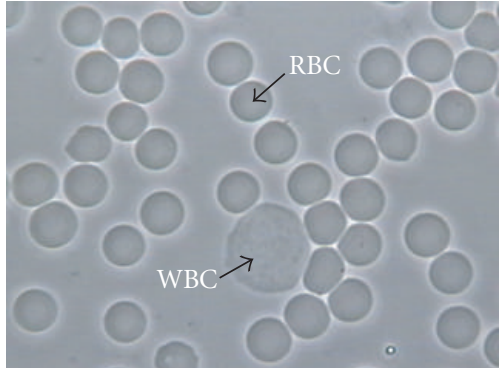

(b)

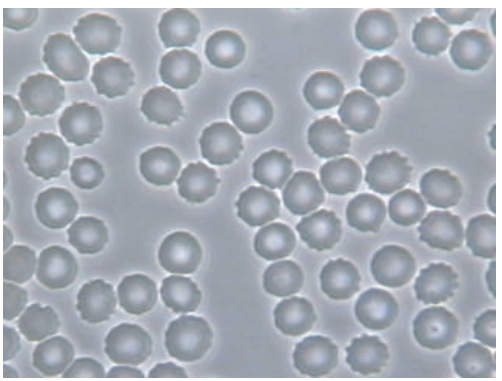

(e)

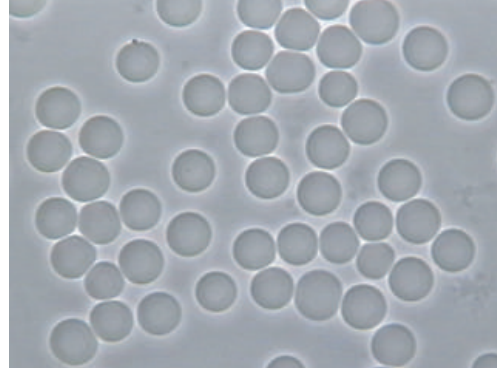

(c)

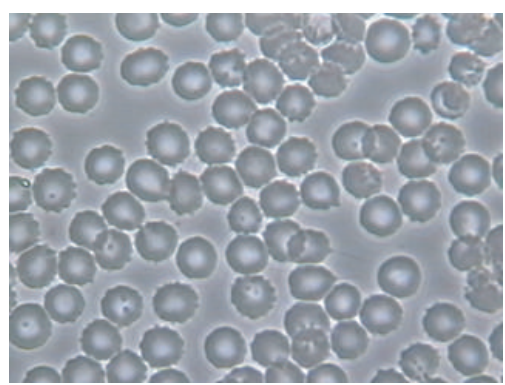

(f)

FIGURE 2: Morphological features of red blood cells after $24 \mathrm{~h}$ of exposure to different concentrations of oxygen: (a) 14.4\%, (b) 20.9\%, (c) $35.5 \%$, (d) $39.8 \%$, (e) $62.5 \%$, and (f) $82.2 \%$. RBC: red blood cell; WBC: white blood cell. Scale bar is $10 \mu \mathrm{m}$.

of oxygen. In our subsequent study, we plan to examine the levels of BAP and antioxidant enzymes, for example, superoxide dismutase, catalase, and glutathione peroxidase, in rats exposed to different concentrations of oxygen for more than $24 \mathrm{~h}$.

Transformed red blood cells, which were induced by oxidative attack from reactive oxygen species, were observed by exposure to $39.8 \%, 62.5 \%$, and $82.2 \%$ oxygen (Figure 2); these findings are consistent with the increased levels of dROMs at these oxygen concentrations (Figure 1(a)). Therefore, we conclude that morphological changes in the red blood cells are linked to increased levels of oxidative stress.

\section{Conclusion}

Exposure to oxygen concentrations higher than $40 \%$ for $24 \mathrm{~h}$ induces excessive levels of oxidative stress.

\section{Conflict of Interests}

The authors declare that they have no conflict of interests.

\section{References}

[1] E. C. Parker, S. S. Survanshi, P. B. Massell, and P. K. Weathersby, "Probabilistic models of the role of oxygen in human decompression sickness," Journal of Applied Physiology, vol. 84, no. 3, pp. 1096-1102, 1998.

[2] A. J. F. Macmillan, "Chapter 3. Sub-atmospheric decompression sickness," in Aviation Medicine, pp. 19-25, Butterworth Heinemann, Oxford, UK, 3rd edition, 1999.
[3] J. F. Kasic, H. M. Smith, and R. I. Gamow, "A self-contained life support system designed for use with a portable hyperbaric chamber," Biomedical Sciences Instrumentation, vol. 25, pp. 7981, 1989.

[4] J. F. Kasic, M. Yaron, R. A. Nicholas, J. A. Lickteig, and R. Roach, "Treatment of acute mountain sickness: hyperbaric versus oxygen therapy," Annals of Emergency Medicine, vol. 20, no. 10, pp. 1109-1112, 1991.

[5] C. Imray, A. Wright, A. Subudhi, and R. Roach, "Acute mountain sickness: pathophysiology, prevention, and treatment," Progress in Cardiovascular Diseases, vol. 52, no. 6, pp. 467-484, 2010.

[6] G. J. Butler, N. Al-Waili, D. V. Passano et al., "Altitude mountain sickness among tourist populations: a review and pathophysiology supporting management with hyperbaric oxygen," Journal of Medical Engineering \& Technology, vol. 35, no. 3-4, pp. 197-207, 2011.

[7] M. A. Austin, K. E. Wills, L. Blizzard, E. H. Walters, and R. Wood-Baker, "Effect of high flow oxygen on mortality in chronic obstructive pulmonary disease patients in prehospital setting: randomised controlled trial," British Medical Journal, vol. 341, p. c5462, 2010.

[8] C. M. Maier and P. H. Chan, "Role of superoxide dismutases in oxidative damage and neurodegenerative disorders," Neuroscientist, vol. 8, no. 4, pp. 323-334, 2002.

[9] K. K. Griendling and G. A. FitzGerald, "Oxidative stress and cardiovascular injury. Part I: basic mechanisms and in vivo monitoring of ROS," Circulation, vol. 108, no. 16, pp. 19121916, 2003.

[10] I. Dalle-Donne, R. Rossi, R. Colombo, D. Giustarini, and A. Milzani, "Biomarkers of oxidative damage in human disease," Clinical Chemistry, vol. 52, no. 4, pp. 601-623, 2006. 
[11] D. Pitocco, F. Zaccardi, E. Di Stasio et al., "Oxidative stress, nitric oxide, and diabetes," Review of Diabetic Studies, vol. 7, no. 1, pp. 15-25, 2010.

[12] A. Alberti, L. Bolognini, D. Macciantelli, and M. Caratelli, "The radical cation of N,N-diethyl-para-phenylendiamine: a possible indicator of oxidative stress in biological samples," Research on Chemical Intermediates, vol. 26, no. 3, pp. 253 $267,2000$.

[13] C. Vassalle, C. Boni, P. Di Cecco, R. Ndreu, and G. C. Zucchelli, "Automation and validation of a fast method for the assessment of in vivo oxidative stress levels," Clinical Chemistry and Laboratory Medicine, vol. 44, no. 11, pp. 1372-1375, 2006.

[14] A. Pasquini, E. Luchetti, V. Marchetti, G. Cardini, and E. L. Iorio, "Analytical performances of d-ROMs test and BAP test in canine plasma. Definition of the normal range in healthy Labrador dogs," Veterinary Research Communications, vol. 32, no. 2, pp. 137-143, 2008.

[15] F. Nagatomo, N. Gu, H. Fujino, I. Takeda, K. Tsuda, and A. Ishihara, "Skeletal muscle characteristics of rats with obesity, diabetes, hypertension, and hyperlipidemia," Journal of Atherosclerosis and Thrombosis, vol. 16, no. 5, pp. 576-585, 2009.

[16] R. Trotti, M. Carratelli, and M. Barbieri, "Performance and clinical application of a new, fast method for the detection of hydroperoxides in serum," Panminerva Medica, vol. 44, no. 1, pp. 37-40, 2002.

[17] S. J. Paralikar and J. H. Paralikar, "High-altitude medicine," Indian Journal of Occupational and Environmental Medicine, vol. 14, no. 1, pp. 6-12, 2010.

[18] N. S. Al-Waili, G. J. Butler, J. Beale et al., "Influences of hyperbaric oxygen on blood pressure, heart rate and blood glucose levels in patients with diabetes mellitus and hypertension," Archives of Medical Research, vol. 37, no. 8, pp. 991-997, 2006.

[19] B. M. Palmquist, B. Philipson, and P. O. Barr, "Nuclear cataract and myopia during hyperbaric oxygen therapy," British Journal of Ophthalmology, vol. 68, no. 2, pp. 113-117, 1984.

[20] F. J. Giblin, V. A. Padgaonkar, V. R. Leverenz et al., "Nuclear light scattering, disulfide formation and membrane damage in lenses of older guinea pigs treated with hyperbaric oxygen," Experimental Eye Research, vol. 60, no. 3, pp. 219-235, 1995.

[21] V. A. Padgaonkar, V. R. Leverenz, K. E. Fowler, V. N. Reddy, and F. J. Giblin, "The effects of hyperbaric oxygen on the crystallins of cultured rabbit lenses: a possible catalytic role for copper," Experimental Eye Research, vol. 71, no. 4, pp. 371-383, 2000.

[22] L. B. Gesell and A. Trott, "De novo cataract development following a standard course of hyperbaric oxygen therapy," Undersea and Hyperbaric Medicine, vol. 34, no. 6, pp. 389-392, 2007.

[23] P. M. Tibbles and J. S. Edelsberg, "Hyperbaric-oxygen therapy," New England Journal of Medicine, vol. 334, no. 25, pp. 1642-1648, 1996.

[24] R. M. Leach, P. J. Rees, and P. Wilmshurst, "ABC of oxygen: hyperbaric oxygen therapy," British Medical Journal, vol. 317, no. 7166, pp. 1140-1143, 1998.

[25] C. K. Narkowicz, J. H. Vial, and P. W. McCartney, "Hyperbaric oxygen therapy increases free radical levels in the blood of humans," Free Radical Research Communications, vol. 19, no. 2, pp. 71-80, 1993.

[26] S. Öter, A. Korkmaz, T. Topal et al., "Correlation between hyperbaric oxygen exposure pressures and oxidative parameters in rat lung, brain, and erythrocytes," Clinical Biochemistry, vol. 38 , no. 8, pp. 706-711, 2005.
[27] S. Öter, T. Topal, S. Sadir et al., "Oxidative stress levels in rats following exposure to oxygen at 3 atm for 0-120 min," Aviation, Space, and Environmental Medicine, vol. 78, no. 12, pp. 1108-1113, 2007.

[28] A. Ishihara, F. Kawano, T. Okiura, F. Morimatsu, and Y. Ohira, "Hyperbaric exposure with high oxygen concentration enhances oxidative capacity of neuromuscular units," Neuroscience Research, vol. 52, no. 2, pp. 146-152, 2005.

[29] A. Matsumoto, T. Okiura, F. Morimatsu, Y. Ohira, and A. Ishihara, "Effects of hyperbaric exposure with high oxygen concentration on the physical activity of developing rats," Developmental Neuroscience, vol. 29, no. 6, pp. 452-459, 2007.

[30] K. Yasuda, N. Aoki, T. Adachi et al., "Hyperbaric exposure with high oxygen concentration inhibits growth-associated increase in the glucose level of diabetic Goto-Kakizaki rats," Diabetes, Obesity and Metabolism, vol. 8, no. 6, pp. 714-715, 2006.

[31] K. Yasuda, T. Adachi, N. Gu et al., "Effects of hyperbaric exposure with high oxygen concentration on glucose and insulin levels and skeletal muscle-fiber properties in diabetic rats," Muscle \& Nerve, vol. 35, no. 3, pp. 337-343, 2007.

[32] A. Matsumoto, F. Nagatomo, K. Yasuda, K. Tsuda, and A. Ishihara, "Hyperbaric exposure with high oxygen concentration improves altered fiber types in the plantaris muscle of diabetic Goto-Kakizaki rats," Journal of Physiological Sciences, vol. 57, no. 2, pp. 133-136, 2007.

[33] N. Gu, F. Nagatomo, H. Fujino, I. Takeda, K. Tsuda, and A. Ishihara, "Hyperbaric oxygen exposure improves blood glucose level and muscle oxidative capacity in rats with type 2 diabetes," Diabetes Technology \& Therapeutics, vol. 12, no. 2, pp. 125-133, 2010.

[34] F. Nagatomo, H. Fujino, I. Takeda, and A. Ishihara, "Effects of hyperbaric oxygenation on blood pressure levels of spontaneously hypertensive rats," Clinical and Experimental Hypertension, vol. 32, no. 3, pp. 193-197, 2010.

[35] F. Nagatomo, N. Gu, H. Fujino et al., "Effects of exposure to hyperbaric oxygen on oxidative stress in rats with type II collagen-induced arthritis," Clinical and Experimental Medicine, vol. 10, no. 1, pp. 7-13, 2010.

[36] T. Nishizaka, F. Nagatomo, H. Fujino et al., "Hyperbaric oxygen exposure reduces age-related decrease in oxidative capacity of the tibialis anterior muscle in mice," Enzyme Research, vol. 2010, Article ID 824763, 2010.

[37] F. Nagatomo, R. R. Roy, H. Takahashi, V. R. Edgerton, and A. Ishihara, "Effect of exposure to hyperbaric oxygen on diabetesinduced cataracts in mice," Journal of Diabetes, vol. 3, no. 4, pp. 301-308, 2011. 


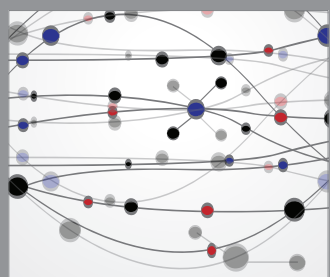

The Scientific World Journal
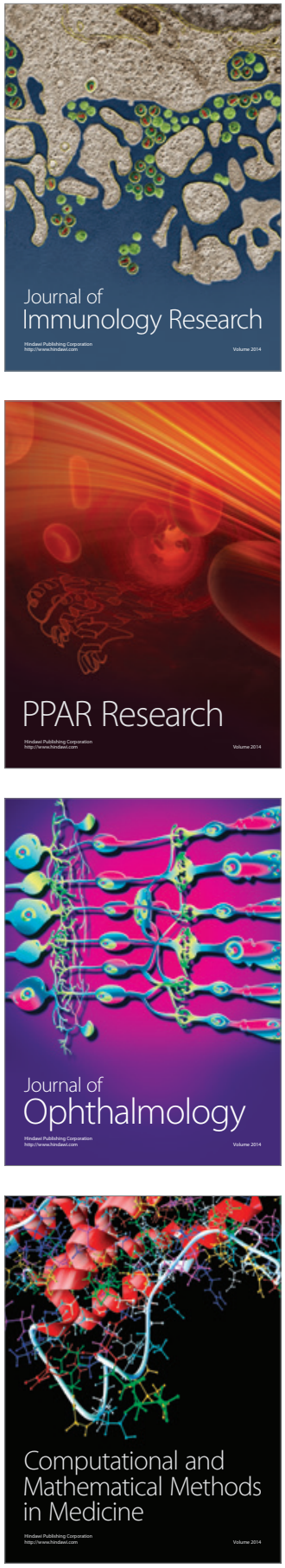

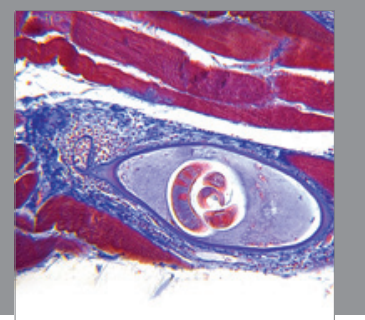

Gastroenterology

Research and Practice
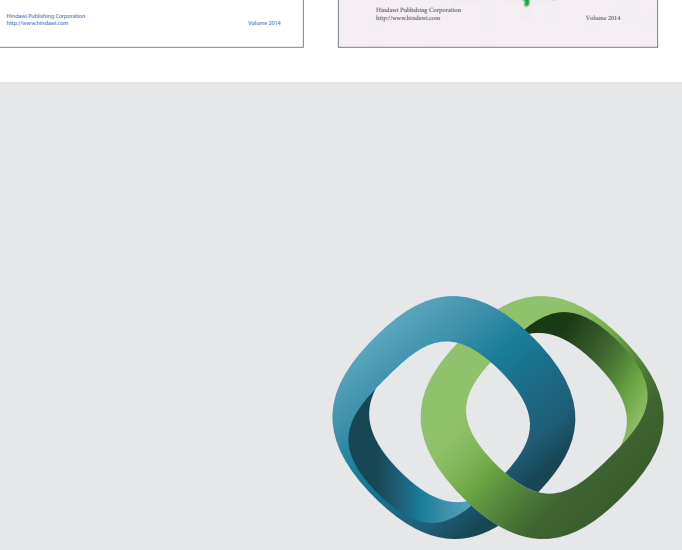

\section{Hindawi}

Submit your manuscripts at

http://www.hindawi.com
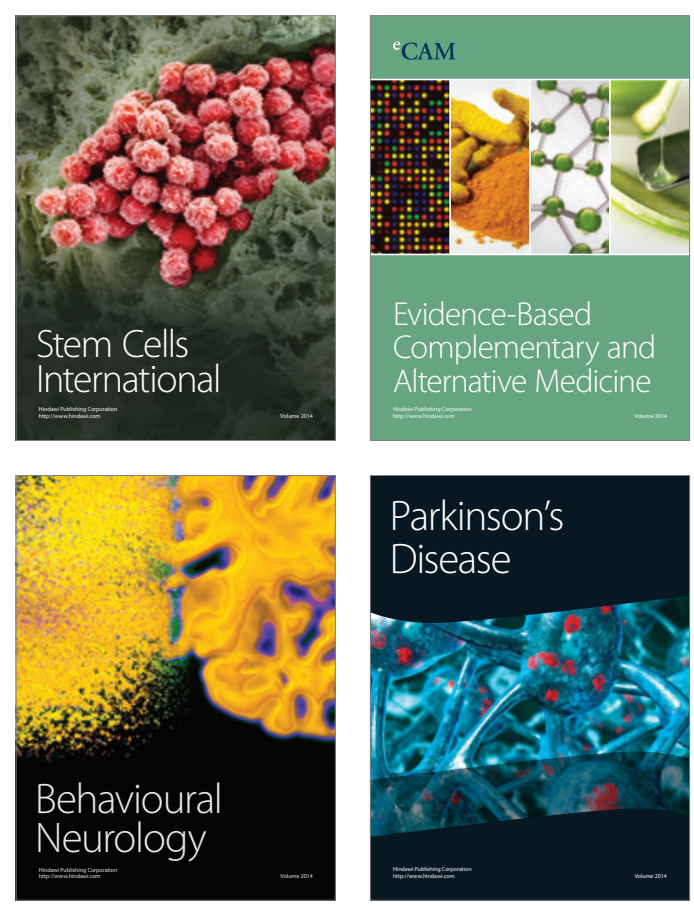

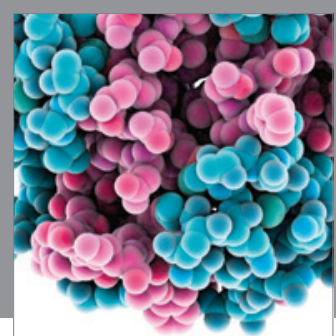

Journal of
Diabetes Research

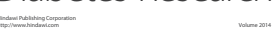

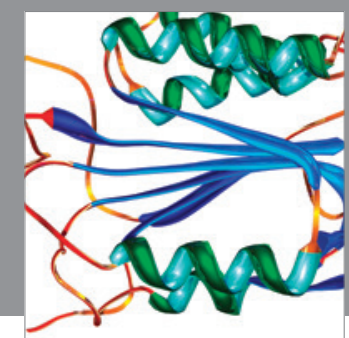

Disease Markers
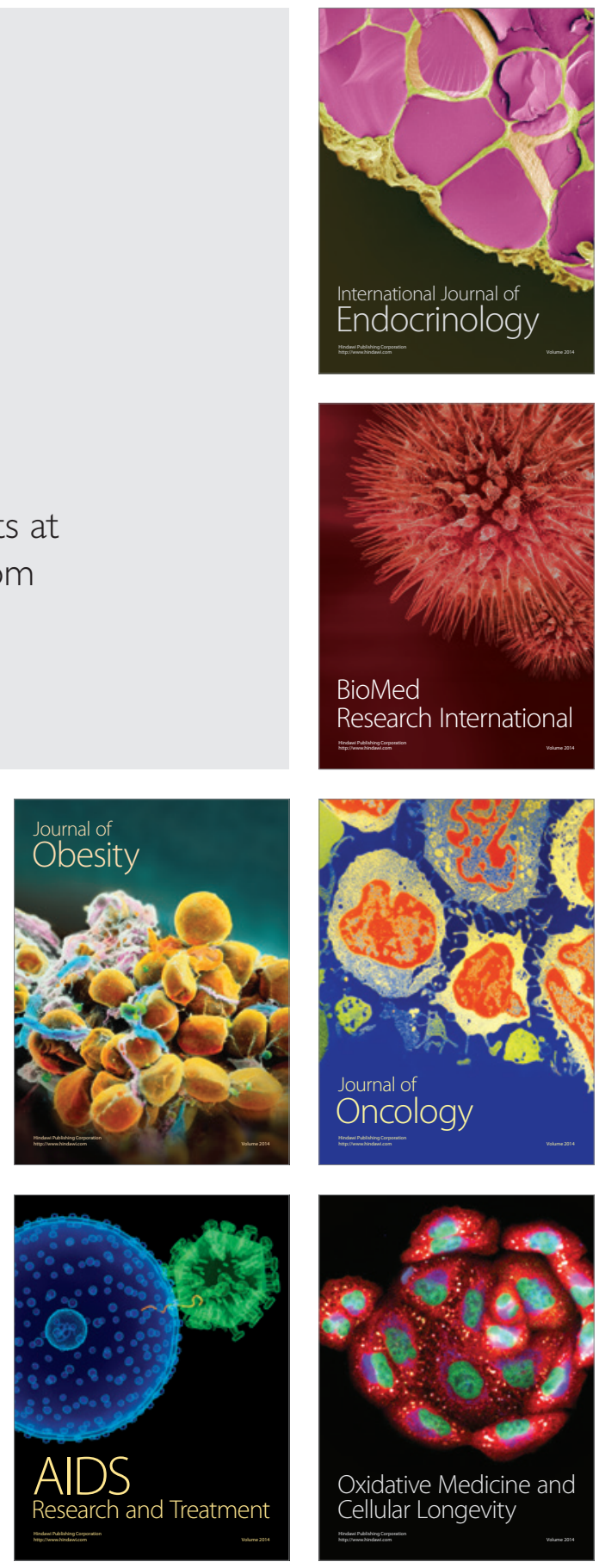